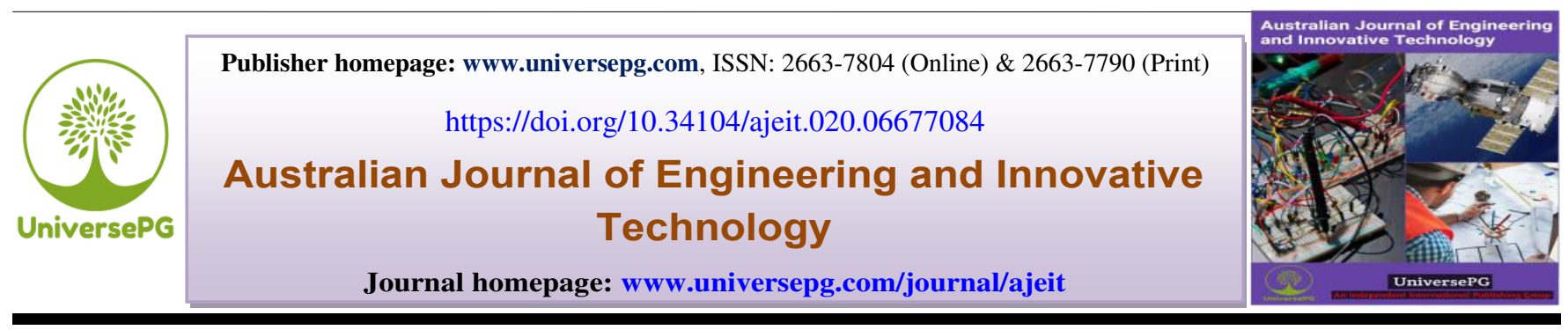

\title{
Study the BER Performance Comparison of MIMO Systems Using BPSK Modulation with ZF and MMSE Equalization
}

\author{
G. M. Waliullah ${ }^{1}$, Diponkor Bala ${ }^{1}$, Ashrafunnahar Hena ${ }^{2}$, Md. Ibrahim Abdullah ${ }^{1}$, and Mohammad \\ Alamgir Hossain $^{1 *}$ \\ ${ }^{1}$ Dept. of Computer Science and Engineering, Islamic University, Kushtia, Bangladesh; and ${ }^{2}$ Dept. of Electrical and \\ Electronic Engineering, Islamic University, Kushtia, Bangladesh. \\ *Correspondence: alamgir@cse.iu.ac.bd (Mohammad Alamgir Hossain, Assistant Professor, Dept. of Computer Science and \\ Engineering, Islamic University, Kushtia, Bangladesh).
}

\begin{abstract}
In the modern age, wireless communication is very helpful in various mobile antenna communication systems. In mobile communication systems, the transmission of data transfer rates is very high and it plays an important role in several services like video, top-quality audio, and mobile integrated service digital network. During the transmission of data at higher data transmission rates through the mobile radio channels, the channel impulse response can spread over many symbol periods as well as causes inter-symbol interference (ISI). Wireless transmission is suffering from fading and interference effects which may be combated with equalizer. Due to fading and interference, it creates a problem for signal recovery in wireless communication. The main objective of this paper is to analyze the different types of equalizers such as ZF and MMSE for BPSK modulation. The simulation result has been developed by using MATLAB toolbox version 2015a and a multi-tap ISI channel is considered. By analyzing the simulation result it shows that if the number of tap lengths is increasing, BER will decrease in ZF equalizer. And finally shows BER vs SNR comparison of two different types of an equalizer and is able to find out MMSE performance is better than ZF equalizer.
\end{abstract}

Keywords: MIMO systems, BPSK modulation, BER performance, ZF, MMSE equalization, AWGN, and SNR.

\section{INTRODUCTION}

We are currently living in the age of wireless technology and day by day the use of wireless technology is growing at a tremendous rate. Multiple Input Multiple Output (MIMO) refers to the antenna configuration technology for wireless transmissions where the multiple antennas are used at both transmitter and receiver sides. The antennas at both ends of the communications circuit are combined to decrease errors and optimize data speed (David et al., 2003; Zhang and Kung, 2003). The MIMO system uses UniversePG I www.universepg.com multiple antennas to transmit multiple parallel signals for transmission. The MIMO techniques have attracted great attention due to multiple antennas at both transmitter and receiver being employed; it provides the possibility of upper data rates compared to single antenna systems (Telatar, 1999; Tarokh et al., 1999). A point-to-point (for single user) MIMO communication system promises large gains for both data transmission rates and reliability and these are accomplished via the utilization of space-time codes (diversity gain oriented) (Ju et al., 2013). 
This technique grants higher channel capacity to wireless systems and is able to increase capacity of the channel linearly with the numerous antennas and link range without additional bandwidth and power requirements (Trimeche et al 2012).

ISI is a one kind of problem in high speed communication. Sometimes ISI can't work perfectly when a transmission interferes with itself and the receiver can't decode the transmission correctly (Theodore, 2002). All types of assumptions consist of AWGN and this type of channel model is not common in the real world. For the lack of frequency spectrum we usually filter the transmitted signal to limit its bandwidth so that we can achieve efficient sharing of frequency response of the channel. Since various workable channels are band-pass and actually, they often respond differently to inputs with different frequency components, i.e. they are dispersive. We need to refine the simple AWGN (or non-dispersive) model so that we can represent this type of practical channel accurately. We only consider the refinement of the dispersive channel mode is,

$$
r(t)=u(t) \otimes h_{c}(t)+n(t)
$$

Where, $\mathrm{u}(\mathrm{t})$ is the transmitted signal, $h_{c}(t)$ is the impulse response of the channel, and $n(t)$ is AWGN with power spectral density $\frac{N_{0}}{2}$.

Wireless communication systems have great advantages and provide higher data rates, Quality of Service (QoS), and link reliability (Ananth, 2012). The modern wireless transmission system has some applications such as IEEE 802.11n (Wi-Fi), 4G, 3GPP Long Term Evolution and WiMAX. The MIMO system uses Multiple Transmit and Multiple Receive antennas which take advantages of multipath propagation during a high distraction environment.

The main objective of this present study is to analyze the different types of equalizers such as ZF and MMSE for BPSK modulation. Then also shows BER vs SNR comparison of two different types of equalizer and try to find out which is the best equalizer of BER for a given SNR (Kumar and Kumar, 2011; Jagan et al., 2010). This paper is designed as follows: Section 2 explains the channel model. Section 3 explains BPSK technique. Section 4 interprets ZF equalizer. Section 5 interprets MMSE equalizer. Section 6 is a simulation model. Section 7 describes simulation results and discussion. And finally the conclusion is drawn in section 8 .

\section{CHANNEL}

Additive White Gaussian Noise is the basic noise model that is used in communication channels for thermal noise. It has some basic ideas:

Additive: the noise is additive, i.e., the received signal is equal to the transmit signal by adding some noise, where the noise is linearly independent of the signal of the channel.

White: the noise is white, i.e., the ability spectral density is flat, therefore the autocorrelation of the noise in time domain is zero for any non-zero time offset.

Gaussian: the noise samples have a normal distribution and the average time domain value is zero. AWGN is usually employed in channel models which are capable of communicating a linear addition of wideband or white noise with a relentless spectral density (expressed as watts per hertz of bandwidth) and therefore the amplitude is Gaussian distribution. The model does not calculate for fading, frequency selectivity, interference and dispersion. Fading is the time variation of the received signal with various variables. However, it produces some simple and obedient mathematical models which are useful for several satellite and deep space communication links. Wideband noise has some natural noise resources: they're thermal variations of atoms in conductors (is called thermal noise), short noise and from Godlike sources like the sun.

\section{a. Transmit Symbol}

Let the transmit symbols be modeled as

$$
S(t)=\sum_{n=-\infty}^{\infty} a_{n} g(t-m T)
$$

Where

$\mathrm{T}$ is the symbol period, $a_{n}$ is the symbol to transmit, $\mathrm{g}(\mathrm{t})$ is the transmit filter, $\mathrm{n}$ is the symbol index and $\mathrm{S}(\mathrm{t})$ is the output waveform. 
Now, let us assume that the transmit pulse shaping filter is not present, i.e, so the transmit symbols can be modeled by the discrete time equivalent

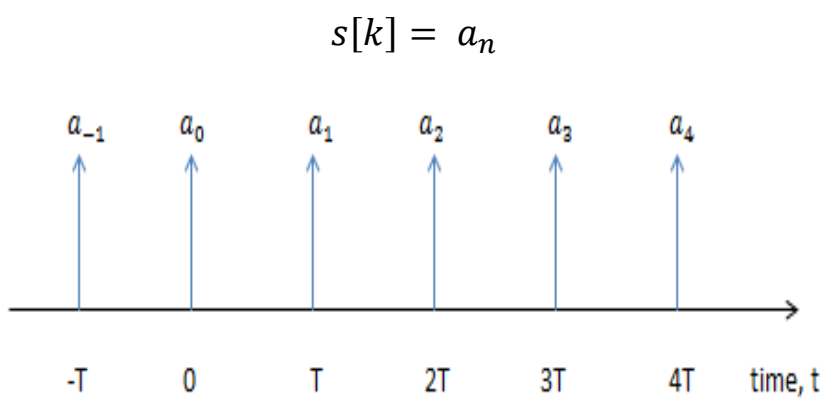

Fig 1: Transmit Symbols.

\section{b. Channel Model}

Consider 3-tap multipath channel with spacing $\mathrm{T}$ i.e.

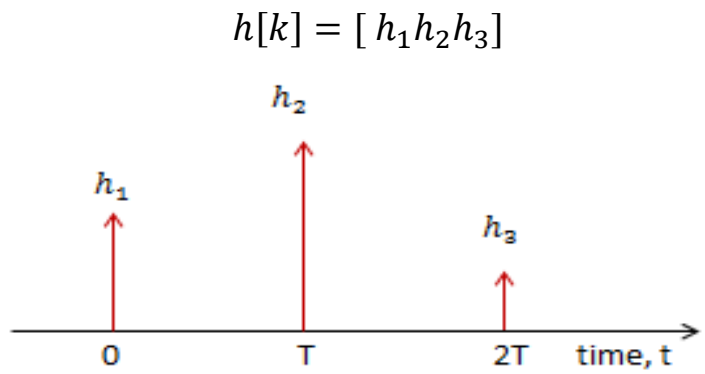

Fig 2: Channel Model (3 tap multipath).

When the received signal falls into corrupted by noise $\mathrm{n}$ as a result of adding a multipath channel then it is known as Additive White Gaussian Noise (AWGN). The value of the noise $n$ follows the Gaussian probability distribution function

$$
p(x)=\frac{1}{\sqrt{2 \pi \sigma^{2}}} e^{\frac{-(x-\mu)^{2}}{2 \sigma^{2}}}
$$

With mean $\mu=0$ and variance $\sigma^{2}=\frac{N_{0}}{2}$.

The received signal is $y[k]=s[k] \otimes h[k]+n$

Where, $\otimes$ is the convolution operator (Gupta et al., 2011).

\section{BINARY PHASE SHIFT KEYING}

BPSK is a one kind of modulation technique which has two phases and it is the simplest form of PSK. It is referred to as 2-PSK. This two phases are separated by $180^{\circ}$ and represented this two phases are $0^{\circ}$ and $180^{\circ}$.

UniversePG I www.universepg.com
Where, $\theta=0^{0}$ represents for binary message 1 , and $\theta=180^{\circ}$ represents for binary message 0

\section{a. Implementation}

The general form of BPSK modulation follows the equation

$$
S_{n}(t)=\sqrt{\frac{2 E_{b}}{T_{b}}} \cos (2 \pi f t+\pi(1-n)), \mathrm{n}=0,1
$$

The two phases are $0^{\circ}$, and $180^{\circ}$. These two phase represents two carrier signals and this are given below

$$
\begin{gathered}
S_{0}(t)=\sqrt{\frac{2 E_{b}}{T_{b}}} \cos (2 \pi f t+\pi) \\
=-\sqrt{\frac{2 E_{b}}{T_{b}}} \cos (2 \pi f t) \text { for binary " } 0 " \\
S_{1}(t)=\sqrt{\frac{2 E_{b}}{T_{b}}} \cos (2 \pi f t) \text { for binary " } 1 "
\end{gathered}
$$

Where, $\mathrm{f}$ is the frequency of the base band, $E_{b}$ is the energy per bit, and $T_{b}$ is the bit duration.

\section{b. Bit Error Rate}

The bit error rate (BER) of BPSK under Additive White Gaussian Noise (AWGN) can be calculated as

$$
P_{b}=Q\left(\sqrt{\frac{2 E_{b}}{N_{0}}}\right)
$$

This is often also the symbol error rate because it represents only one bit per symbol.

\section{ZERO FORCING EQUALIZER}

Zero Forcing equalizer is a method that applies inversely to the received signal so that it can restore the signal. It is a one kind of graphical equalization algorithm that is widely used in communication systems. This form of equalizer was first proposed by Robert Lucky.

It has many useful applications. For example, by studying deeply of the MIMO system in 802.11n to know the accurate information about the channel at every antenna and two or more streams are recovered from the receive signal. By applying ZF equalizer technique it will be possible to put down the value of Inter Symbol Interference (ISI) at zero for a noise free channel (Islam et al., 2020). This will be useful when 
ISI is significant compared to noise. The Zero Forcing Equalizer $C(f)$ is represented by $C(f)=1 / F(f)$ where $f$ is a channel frequency response. The combination of channel and equalizer presents a flat frequency response and linear phase $\mathrm{F}(\mathrm{f}) \mathrm{C}(\mathrm{f})=1$ (Ju et al., 2013; Kumar and Piyush, 2017)

\section{a. Zero Forcing(ZF) Equalizer for $2 \times 2$ MIMO channel}

Consider a $2 \times 2$ MIMO channel, the received signal on the first receive antenna is,

$$
y_{1}=h_{1,1} x_{1}+h_{1,2} x_{2}+n_{1}=\left[h_{1,1} h_{1,2}\right]\left[\begin{array}{l}
x_{1} \\
x_{2}
\end{array}\right]+n_{1}
$$

The received signal on the second receive antenna is,

$$
y_{2}=h_{2,1} x_{1}+h_{2,2} x_{2}+n_{2}=\left[h_{2,1} h_{2,2}\right]\left[\begin{array}{l}
x_{1} \\
x_{2}
\end{array}\right]+n_{2}
$$

Where,

$y_{1}, y_{2}$ are the received symbol on the first and second antenna respectively,

$h_{1,1}$ is the channel where 1 is the both $1^{\text {st }}$ sending antenna and $1^{\text {st }}$ receive antenna,

$h_{1,2}$ is the channel where 2 is the $2^{\text {nd }}$ sending antenna and 1 is the $1^{\text {st }}$ receive antenna,

$h_{2,1}$ is the channel where 1 is the $1^{\text {st }}$ sending antenna and 2 is the $2^{\text {nd }}$ receive antenna,

$h_{2,2}$ is the channel where 2 is the both $2^{\text {nd }}$ transmit antenna and $2^{\text {nd }}$ receive antenna,

$x_{1}, x_{2}$ are the sending symbols, and

$n_{1}, n_{2}$ is the noise on $1^{\text {st }}, 2^{\text {nd }}$ receive antennas.

The equation can be illustrated in matrix notation as follows:

$$
\left[\begin{array}{c}
y_{1} \\
y_{2}
\end{array}\right]=\left[\begin{array}{ll}
h_{1,1} & h_{1,2} \\
h_{2,1} & h_{2,2}
\end{array}\right]\left[\begin{array}{c}
x_{1} \\
x_{2}
\end{array}\right]+\left[\begin{array}{c}
n_{1} \\
n_{2}
\end{array}\right]
$$

Equivalently,

$$
Y=H X+N
$$

Where,

$\mathrm{Y}=$ Received Symbol Matrix, $\mathrm{H}=$ Channel matrix, $\mathrm{X}$ $=$ Transmitted symbol Matrix, and $\mathrm{N}=$ Noise Matrix.

To solve for $x$, we need to find a matrix $W$ which satisfies $\mathrm{WH}=\mathrm{I}$. The Zero Forcing $(\mathrm{ZF})$ detector for meeting this constraint is given by,

$$
W=\left(H^{H} H\right)^{-1} H^{H}
$$

Where,

W - Equalization Matrix, and H - Channel Matrix.

UniversePG I www.universepg.com
This matrix is known as the Pseudo inverse for a general $\mathrm{m} x \mathrm{n}$ matrix where

$$
\begin{array}{r}
H^{H} H=\left[\begin{array}{ll}
h_{1,1}^{*} & h_{2,1}^{*} \\
h_{1,2}^{*} & h_{2,2}^{*}
\end{array}\right]\left[\begin{array}{ll}
h_{1,1} & h_{1,2} \\
h_{2,1} & h_{2,2}
\end{array}\right] \\
=\left[\begin{array}{cc}
\left|h_{1,1}\right|^{2}+\left|h_{2,1}\right|^{2} & h_{1,1}^{*} h_{1,2}+h_{2,1}^{*} h_{2,2} \\
h_{1,2}^{*} h_{1,1}+h_{2,2}^{*} h_{2,1} & \left|h_{1,2}\right|^{2}+\left|h_{2,2}\right|^{2}
\end{array}\right]
\end{array}
$$

Furthermore, it can be seen that, following zero forcing equalization, the channel for symbol transmitted from each spatial dimension (space is antenna) is a like a $1 \times 1$ Rayleigh fading channel. Hence the BER for $2 \times 2$ and $4 \times 4$ MIMO channel in Rayleigh fading with Zero Forcing equalization is similar to the BER obtained for a $1 \times 1$ channel in Rayleigh fading (Prasad and Varanasi, 2004).

For BPSK modulation in Rayleigh fading channel, the bit error rate is derived as,

$$
P_{b}=\frac{1}{2}\left(1-\sqrt{\frac{\frac{E_{b}}{N_{o}}}{\left(\frac{E_{b}}{N_{o}}\right)+1}}\right)
$$

Where,

$P_{b}$ - Bit Error Rate, and $\frac{E_{b}}{N_{o}}$ - Signal to noise Ratio.

\section{MINIMUM MEAN SQUARE ERROR EQUA- LIZER}

A Minimum Mean Square Error (MMSE) is a conventional way that calculates the Mean Square Error (MSE) and tries to minimize the error. For this reason it refers to the best common measure estimator quality. The main property of MMSE is that it fails to remove all ISI perfectly but decreases the total power of the noise and ISI components in the output. Let $x$ be an unknown random variable, and let $y$ be a known random variable. An estimator $\mathrm{x}^{\wedge}(\mathrm{y})$ is any function of the measurement $y$, and its mean square error is given by

$$
M S E=E\left\{\left(X^{\wedge} X^{2}\right)\right\}
$$

Where; the expectation is taken over both $\mathrm{x}$, and $\mathrm{y}$.

By following the term $\mathrm{AY}+\mathrm{b}$ we acquire a minimum MSE overall estimator and it is known as linear MMSE estimator. Where $\mathrm{Y}$ is a random vector, $\mathrm{A}$ is a matrix and $\mathrm{b}$ is a vector. 


\section{a. Minimum Mean Square Error Equalizer for $2 \times 2$ MIMO channel}

Consider a $2 \times 2$ MIMO channel, the received signal on the first receive antenna is,

$$
y_{1}=h_{1,1} x_{1}+h_{1,2} x_{2}+n_{1}=\left[h_{1,1} h_{1,2}\right]\left[\begin{array}{l}
x_{1} \\
x_{2}
\end{array}\right]+n_{1}
$$

The received signal on the second receive antenna is,

$$
y_{2}=h_{2,1} x_{1}+h_{2,2} x_{2}+n_{2}=\left[h_{2,1} h_{2,2}\right]\left[\begin{array}{l}
x_{1} \\
x_{2}
\end{array}\right]+n_{2}
$$

Where,

$y_{1}, y_{2}$ are the received symbol on the first and second antenna respectively,

$h_{1,1}$ is the channel where 1 is the both $1^{\text {st }}$ sending antenna and $1^{\text {st }}$ receive antenna,

$h_{1,2}$ is the channel where 2 is the $2^{\text {nd }}$ sending antenna and 1 is the $1^{\text {st }}$ receive antenna,

$h_{2,1}$ is the channel where 1 is the $1^{\text {st }}$ sending antenna and 2 is the $2^{\text {nd }}$ receive antenna,

$h_{2,2}$ is the channel where 2 is the both $2^{\text {nd }}$ sending antenna and $2^{\text {nd }}$ receive antenna,

$x_{1}, x_{2}$ are the sending symbols, and

$n_{1}, n_{2}$ is the noise on $1^{\text {st }}, 2^{\text {nd }}$ receive antennas.

The equation can be illustrated in matrix notation as follows:

$$
\left[\begin{array}{l}
y_{1} \\
y_{2}
\end{array}\right]=\left[\begin{array}{ll}
h_{1,1} & h_{1,2} \\
h_{2,1} & h_{2,2}
\end{array}\right]\left[\begin{array}{c}
x_{1} \\
x_{2}
\end{array}\right]+\left[\begin{array}{c}
n_{1} \\
n_{2}
\end{array}\right]
$$

Equivalently,

$$
Y=H X+N
$$

Where,

$\mathrm{Y}=$ Received Symbol Matrix, $\mathrm{H}=$ Channel matrix, $\mathrm{X}$ $=$ Transmitted symbol Matrix, and $\mathrm{N}=$ Noise Matrix.

The Minimum Mean Square Error (MMSE) approach tries to find a coefficient $W$ which minimizes the criterion,

$$
E\left\{\left[W_{y-x}\right]\left[W_{y-x}\right] H\right\}
$$

Where,

W - Equalization Matrix, H - Channel Matrix, n Channel noise, and y - Received signal.

To solve for $x$, we need to find a matrix $W$ which satisfies $W H=I$. The Minimum Mean Square Error
(MMSE) detector for meeting this constraint is given by,

$$
W=\left[H^{H} H+N_{o} I\right]^{-1} H^{H}
$$

This matrix is known as the Pseudo inverse for a general $\mathrm{m} x \mathrm{n}$ matrix where

$$
\begin{array}{r}
H^{H} H=\left[\begin{array}{ll}
h_{1,1}^{*} & h_{2,1}^{*} \\
h_{1,2}^{*} & h_{2,2}^{*}
\end{array}\right]\left[\begin{array}{ll}
h_{1,1} & h_{1,2} \\
h_{2,1} & h_{2,2}
\end{array}\right] \\
=\left[\begin{array}{cc}
\left|h_{1,1}\right|^{2}+\left|h_{2,1}\right|^{2} & h_{1,1}^{*} h_{1,2}+h_{2,1}^{*} h_{2,2} \\
h_{1,2}^{*} h_{1,1}+h_{2,2}^{*} h_{2,1} & \left|h_{1,2}\right|^{2}+\left|h_{2,2}\right|^{2}
\end{array}\right]
\end{array}
$$

When comparing two equations i.e. eq.(13) and eq.(22) in Zero Forcing Equalizer, we see that both equations are comparable except $N_{o} I$.Actually, when the value of the noise term is zero, the MMSE equalizer minimizes to Zero Forcing equalizer.

\section{SIMULATION MODEL}

The following steps were taken to design the system -

(a) Random binary sequence is generated.

(b) Applying BPSK modulation where bit 0 represented as -1 and bit 1 represented as +1 .

(c) Now convolving the symbol with a multipath channel.

(d) White Gaussian Noise is added.

(e) Now two different programs are prepared -

1. First zero forcing equalization technique is applied for varying tap length and BER is calculated (Islam and Hossain, 2019). Then simulated BER value is compared. Then demodulated the received data and count no. of bit errors for repeating values of $\frac{E_{b}}{N_{0}}$.

2. Second we performed MMSE equalization technique for different tap-lengths and calculated BER and compared BER values between two equalizers namely MMSE and ZF. Finally, simulated value is done.

\section{RESULT AND DISCUSSION:}

This section provides a summarized interpretation and comparative analysis for signal detection using BPSK modulation in ZF and MMSE equalizer. All simulation processes developed in the MATLAB environment. 
As shown in Fig 1, this is the analysis for zero forcing equalizer for various tap lengths. From the graph it is noticed that if the number of tap lengths are increasing, the bit error rate will decrease. Increasing the equalizer tap length from 3 to 5 showed feasible performances developed but when the tap length is 9, then BER decreases than any other tap length. Furthermore, it is seen that there is a lot of gap between theoretical and practical BER.

\section{a. Bit Error Rate probability curve for BPSK modulation in ISI with ZF equalizer}

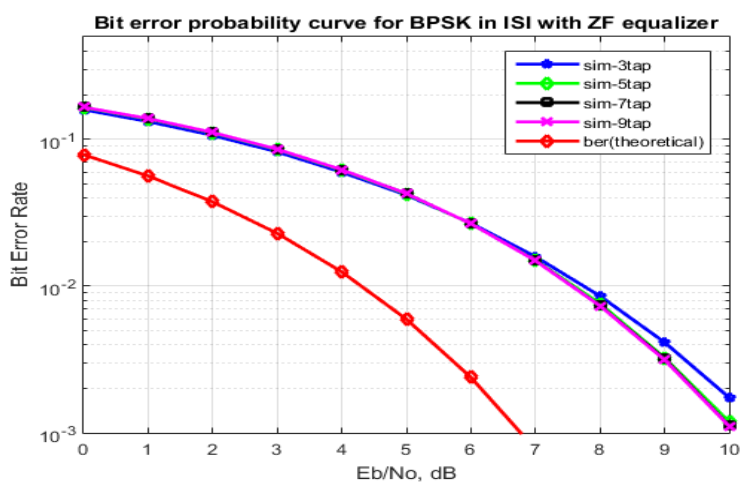

Fig 3: Bit error probability curve for BPSK in ISI with ZF equalizer.

\section{b. Comparison BER curve between $\mathrm{ZF}$ and MMSE equalizer}

This is the result of a comparison bit error probability curve between Zero Forcing and Minimum Mean Square Error equalizer as shown in Fig 2. It can be seen from Fig 2 that bit error rate value of MMSE has decreased than ZF equalizer for fixed tap length. But there is a gap between theoretical and practical BER. So MMSE detectors are more efficient than ZF.

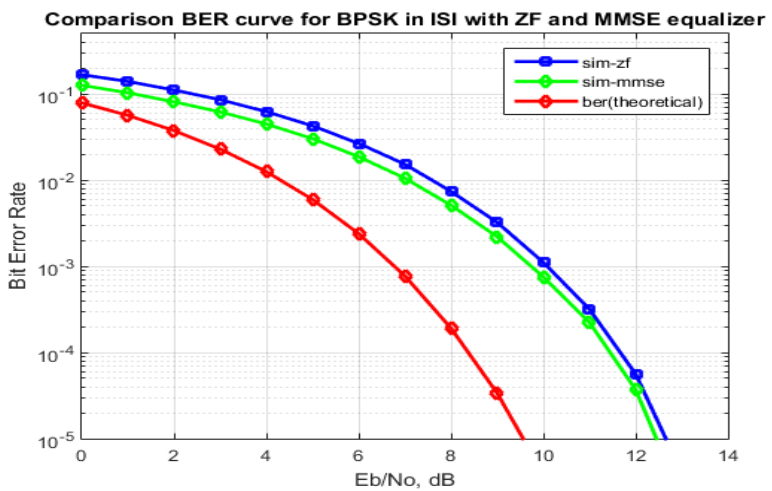

Fig 4: Comparison BER curve for BPSK modulation with ZF and MMSE equalizer.

\section{c. Comparison BER values between $\mathrm{ZF}$ and MMSE equalizer using BPSK modulation}

In the following Table 1 shows Bit Error Rate (BER) values comparison between $\mathrm{ZF}$ and MMSE equalizer. This table analysis shows that the simulated bit error rate (simBer) value of MMSE is much smaller than the $\mathrm{ZF}$ equalizer. When the SNR value is gradually increased, the BER value decreases and finally when the SNR value reaches 13, 14 and 15 then the BER value remains to 0 .

Table 1: BER performance comparison between ZF and MMSE using BPSK modulation.

\begin{tabular}{|c|c|c|}
\hline SNR (dB) Value & \multicolumn{2}{|c|}{ BER Value } \\
\hline & simBer_ZF & simBer_MMSE \\
\hline 0 & 0.1664 & 0.1252 \\
\hline 1 & 0.1383 & 0.1031 \\
\hline 2 & 0.1113 & 0.0814 \\
\hline 3 & 0.0855 & 0.0617 \\
\hline 4 & 0.0623 & 0.0444 \\
\hline 5 & 0.0423 & 0.0297 \\
\hline 6 & 0.0264 & 0.0184 \\
\hline 7 & 0.0148 & 0.0102 \\
\hline 8 & 0.0075 & 0.0050 \\
\hline 9 & 0.0031 & 0.0020 \\
\hline 10 & 0.0011 & 0.0006 \\
\hline 11 & 0.0003 & 0.0001 \\
\hline 12 & 0.0001 & 0.0000 \\
\hline 13 & 0.0000 & 0.0000 \\
\hline 14 & 0 & 0 \\
\hline 15 & 0 & 0 \\
\hline
\end{tabular}

Based on the simulation results analyzed so far, it is concluded that MMSE is more accurate and suits a wide range of channel state conditions expressed in SNR ratio. The zero forcing method is an approximate method more suitable for high signal to noise ratio.

The MMSE is working well for all signals to noise ratios but it needs huge computational effort.

\section{CONCLUSION:}

This paper presents the particular information about the Zero Forcing (ZF) and Minimum Mean Square Error (MMSE) Equalizer as well as show the comparison of BER performance between them using 
BPSK modulation. All the simulation results will be shown about the Bit Error Rate (BER) features for the both types of equalizers. This paper uses the MATLAB framework for the analysis of simulation. Based on the simulation results we learn that as the tap length increases, BER decreases with Zero Forcing (ZF) equalization for noise free channel. On the other hand, when we compare both equalization techniques it is seen that the BER value of MMSE is decreased for the AWGN channel than ZF equalizer. When SNR value is serially high the BER value gradually decreases and finally reaches the value is 0 . $\mathrm{ZF}$ equalizer enhances the noise in the channel while the MMSE equalizer provides better noise immunity and removes a marginal noise. Furthermore, Zero Forcing and MMSE equalizers were simulated and the results compared. Finally, the results show that MMSE equalizer performs better than $\mathrm{ZF}$ equalizer.

\section{ACKNOWLEDGEMENTS:}

First of all, I must thank the Almighty upon who rests the supreme authority. I would like to express my heartfelt gratitude to the authority of Islamic University to the successful research Work.

\section{CONFLICT OF INTERESTS:}

The author (s) declared no potential conflicts of the interest to publish it.

\section{REFERENCES:}

1. Ananth, A.. (2012). "Study Of Transmission Characteristics Of 2x2 Mimo System for OFDM Multiplexing and Bpsk Modulation With ZF Equalizer And MMSE Receivers." https://www.semanticscholar.org/paper/StudyOf-Transmission-Characteristics-Of-2x2-MimoAnanth/e6d7123ee10a5b5d5af3c2addaf811e7bc 831520\#citing-papers

2. David Gesbert, Mansoor Shafi, Danshan Shiv, and Smith, P.J. (2003). "From theory to practice: An over View of MIMO space time coded wireless system," IEEE Journal on selected areas in Communication, 21(3), pp. 281- 301.

https://doi.org/10.1109/JSAC.2003.809458

3. Gupta, Megha, et al. (2011). "Bit Error Rate Performance in OFDM System Using MMSE

UniversePG I www.universepg.com
MLSE Equalizer Over Rayleigh Fading Channel Through The BPSK, QPSK, 4QAM \& 16- QAM Modulation Technique." International Journal of Engineering Research and Applications (IJERA), 1(3): pp. 1005-1011.

http://www.ijera.com/papers/vol\%201\%20issue \%203/XL01310051011.pdf

4. Islam T., Akter N. and Jahan N. (2020). MHD free convective heat transfer in a triangular enclosure filled with Copper-water nanofluid, Int. J. Mat. Math. Sci., 2(2), pp. 29-38. https://doi.org/10.34104/ijmms.020.029038

5. Islam MT and Hossain MS. (2019). Hybridization of vigenere technique with the collaboration of RSA for secure communication, Aust. J. Eng. Innov. Technol., 1(6), pp. 6-13.

https://doi.org/10.34104/ajeit.019.06013

6. Jagan Naveen V., Murali Krishna K., and Raja Rajeswari K., (2010). "Performance analysis of equalization techniques for MIMO systems in wireless communication," International Journalof Smart Home, 4(4), pp. 47-63. https://www.earticle.net/Article/A148075

7. Ju, Meiyan, et al. (2013). "Comparison of multiuser MIMO systems with MF, ZF and MMSE receivers." IEEE Third International Conference on Information Science and Technology (ICIST). IEEE, 2013.

8. Kumar, Arun, and Piyush Vardhan, (2017). "Design, Simulation \& Concept Verification of $4 \times 4,8 \times 8$ MIMO With ZF, MMSE and BF Detection Schemes." Electrical, Control and Communication Engineering, 13(1): pp. 6974.

https://doi.org/10.1515/ecce-2017-0010

9. Kumar Nagarajan Sathish, K. R. Shankar Kumar, (2011). "Bit Error Rate Performance Analysis of ZF, ML and MMSE Equalizers for MIMO Wireless Communication Receiver. "EuropeanJournal of Scientific Research, 59(4), pp. 522-532.

http://read.pudn.com/downloads602/doc/comm/ 2457270/x3.pdf

10. Prasad N. and Varanasi M. K. (2004). "Analysis of decision feedback detection for 
MIMO rayleigh fading channels and the optimization of rate and power allocations," IEEE Trans. Inf. Theory, 50, pp. 1009-1025.

11. Tarokh V., Naguiband A. et al. (1999). "Space- time codes for high data rate Wireless communication: performance criteria in the presence of channel estimation errors, mobility, and Multiple paths," IEEE Transactions on Communications, 47(2), pp. 199-207.

12. Telatar I. E., (1999). "Capacity of multiantennaGaussian channels," Eur. Trans. Telecommun., 10(6), pp. 585-595. https://doi.org/10.1002/ett.4460100604
13. Theodore. S. Rappaport, (2002). "Wireless Communication, $2^{\text {nd }}$ Edition, PHI, 2002'. https://www.amazon.com/Wireless-Communic ations-Principles-Practice-2nd/dp/0130422320

14. Trimeche, Abdessalem, et al. (2012). "Performance analysis of ZF and MMSE equalizers for MIMO systems." $7^{\text {th }}$ International Conference on Design \& Technology of Integrated Systems in the Nanoscale Era. IEEE, 2012.

15. Zhang, X. and Kung, S., (2003). "Capacity analysis for parallel and sequential MIMO equalizers," IEEE Transactions on Signal Processing, 51, pp. 2989-3002. https://doi.org/10.1109/TSP.2003.818151 